\title{
Interaction potential for water dimer from symmetry-adapted perturbation theory based on density functional description of monomers
}

\author{
Robert Bukowski and Krzysztof Szalewicz ${ }^{\text {a) }}$ \\ Department of Physics and Astronomy, University of Delaware, Newark, Delaware 19716

\begin{abstract}
Gerrit Groenenboom and Ad van der Avoird
Institute of Theoretical Chemistry, NSR-Center, University of Nijmegen, Toernooiveld, 6525 ED Nijmegen, The Netherlands
\end{abstract}

(Received 8 May 2006; accepted 12 June 2006; published online 24 July 2006)

\begin{abstract}
A new six-dimensional interaction potential for the water dimer has been obtained by fitting interaction energies computed at 2510 geometries using a variant of symmetry-adapted perturbation theory (SAPT) based on density functional theory (DFT) description of monomers, referred to as SAPT(DFT). The stationary points, second virial coefficient, vibration-rotation-tunneling spectrum, and structure of liquid water computed with the new potential are in very good agreement with experiment and advanced $a b$ initio calculations, confirming the high level of accuracy provided by SAPT(DFT). @ 2006 American Institute of Physics. [DOI: 10.1063/1.2220040]
\end{abstract}

\section{INTRODUCTION}

Computational treatment of weak intermolecular interactions, also referred to as van der Waals interactions, requires the use of methods capable of handling long-range electron correlation effects, such as the dispersion interaction. Since such effects cannot be properly captured by the density functional theory (DFT), at least in its current form, one usually needs to resort to more time-consuming methods, based on a wave function formalism. In recent years, a new method has been developed, which takes advantage of the ability of DFT to describe electron correlation at the short range-within the isolated monomers-but treats the long-range van der Waals effects using the formalism of symmetry-adapted perturbation theory (SAPT). The starting point of this approach is Kohn-Sham (KS) determinants as zeroth-order monomer wave functions and it is developed up to the second order in the intermolecular interaction operator. This approach, referred to as SAPT(DFT), naturally leads to a decomposition of the total interaction energy into physically interpretable terms: the electrostatic, induction, exchange, and dispersion energies. The dispersion energy is potentially exact in SAPT(DFT), which distinguishes this method from other DFTbased approaches and from various proposed extensions of currently used exchange-correlation functionals.

Originally proposed by Williams and Chabalowski, ${ }^{1}$ SAPT(DFT) has been developed by Misquitta $e t$ al. ${ }^{2,3}$ and, independently, by Hesselmann and Jansen. ${ }^{4-6}$ In more recent work, ${ }^{7,8}$ time-saving density-fitting techniques have been introduced to extend the range of applicability of the method to larger systems, allowing calculations for dimers of biological or industrial interest, such as the RDX dimer, ${ }^{8,9}$ with monomers consisting of about 20 atoms. With large molecules in

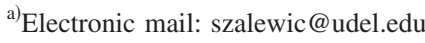

mind, it is imperative to verify the performance of SAPT(DFT) using model systems, for which benchmark results are known. Such tests, involving single-point calculations for small dimers, were carried out in Refs. 10 and 11 and showed that the individual interaction components from SAPT(DFT) are often more accurate than the standard SAPT at the currently programed level.

In this paper, we further test the accuracy of SAPT(DFT) by computing the complete, six-dimensional interaction potential energy surface (PES) for the water dimer and testing it against a set of experimentally measured observables as well as the results of high-level $a b$ initio calculations. In addition to testing the SAPT(DFT) method, our work provides a new two-body potential for water. This potential increases our knowledge of the interactions between water molecules, crucial for our understanding of the peculiar properties of water, such as rich spectra of water clusters or intricacies of structure and thermodynamics of liquid water and ice. An accurate dimer interaction potential is also a necessary ingredient for fundamental investigations of the role of nonadditive interactions in water. Theoretical studies of these issues are too numerous to be listed here. Of special interest for the current work is the $a b$ initio potential SAPT- $5 s,{ }^{12}$ shown in Ref. 13 to almost quantitatively reproduce the vibration-rotationtunneling (VRT) spectrum of water dimer. Despite the confirmed spectroscopic quality of SAPT- $5 s$, the structure of liquid water obtained from Monte Carlo (MC) simulations (with the nonadditive interactions properly included) was less satisfactory. ${ }^{14}$ The new dimer potential obtained in the present work using the SAPT(DFT) method will allow to reexamine this problem in a new light.

\section{METHOD}

The SAPT(DFT) interaction energy is expressed as a sum of perturbative corrections, 


$$
\begin{aligned}
E_{\mathrm{int}}^{\mathrm{SAPT}(\mathrm{DFT})}= & E_{\mathrm{elst}}^{(1)}(\mathrm{KS})+E_{\mathrm{exch}}^{(1)}(\mathrm{KS})+E_{\mathrm{ind}}^{(2)}(\mathrm{CKS}) \\
& +\widetilde{E}_{\text {exch-ind }}^{(2)}(\mathrm{CKS})+E_{\text {disp }}^{(2)}(\mathrm{CKS}) \\
& +\widetilde{E}_{\text {exch-disp }}^{(2)}(\mathrm{CKS})+\delta E_{\mathrm{int}}^{\mathrm{HF}} .
\end{aligned}
$$

All the components in Eq. (1), the detailed definitions of which are given in Ref. 11, have a clear physical interpretation. The electrostatic term, $E_{\text {elst }}^{(1)}(\mathrm{KS})$, describes Coulomb interactions of KS charge densities of the unperturbed monomers. The superscript "(1)" emphasizes that this term is of the first order in the intermolecular interaction operator. The second-order induction energy $E_{\text {ind }}^{(2)}(\mathrm{CKS})$ corresponds to the classical interaction of the permanent density of one monomer with the induced density of the other, while the dispersion energy $E_{\text {disp }}^{(2)}(\mathrm{CKS})$ represents the energy lowering due to the correlation between the instantaneous fluctuations of monomer densities. Both these corrections are computed using frequency-dependent density susceptibility (FDDS) functions (propagators) obtained from coupled Kohn-Sham (CKS) calculations. Equation (1) also contains the first- and second-order exchange corrections, resulting from the imposition of Pauli exclusion principle and responsible for the repulsive wall of the interaction potential. The quantities denoted with a tilde are obtained approximately as scaled estimates. ${ }^{11}$ In the case of polar systems, such as water, it is also necessary to include the term $\delta E_{\text {int }}^{\mathrm{HF}}$, collecting higherorder induction and exchange-induction effects present in the supermolecular Hartree-Fock (HF) interaction energy.

All SAPT(DFT) calculations have been performed using the extended monomer-centered basis set $\left(\mathrm{MC}^{+} \mathrm{BS}\right.$, with the plus indicating the extension) of Ref. 15, consisting of the $5 s 3 p 2 d 1 f / 3 s 2 p$ sets on oxygen/hydrogen atoms and an additional $3 s 2 p 1 d$ set of bond functions. The monomers were described with the PBE0 functional ${ }^{16,17}$ asymptotically corrected using the Fermi-Amaldi scheme. ${ }^{18,19}$ The ionization potential needed to define the asymptotic correction was taken as $0.4638 \mathrm{~h}^{20}$ The CADPAC program ${ }^{21}$ was used to solve the KS equations for the monomers and to produce the matrix elements needed in the calculation of the CKS propagators. The geometry of each water molecule was fixed with O-H distances equal to $0.9716257 \AA$ and the $\mathrm{HOH}$ angle equal to $104.66^{\circ}$, as in Ref. 15 , both resulting from averaging over the ground vibrational state of the molecule. The set of 2510 dimer geometries for which the interaction energies were calculated is the same as the one used in Ref. 12 to obtain the SAPT-5s potential. This set exhaustively covers characteristic points on the potential surface, regions important for the second virial coefficient, as well as geometries characteristic for liquid water and ice. The values of $\delta E_{\mathrm{int}}^{\mathrm{HF}}$ were taken directly from the work of Refs. 12 and 15.

The interaction energies $E_{\text {int }}^{\mathrm{SAPT}(\mathrm{DFT})}$ calculated as in Eq. (1) were fitted to the same site-site functional form as that used in Ref. 12,

$$
\begin{aligned}
V= & \sum_{a b}\left[\left(1+\sum_{m=1}^{3} a_{m}^{a b} r_{a b}^{m}\right) \mathrm{e}^{\alpha_{a b}-\beta_{a b} r_{a b}+f_{1}\left(\delta_{1}^{a b}, r_{a b}\right) \frac{q_{a} q_{b}}{r_{a b}}}\right. \\
& \left.+\sum_{n=6,8,10} f_{n}\left(\delta_{n}^{a b}, r_{a b}\right) \frac{C_{n}^{a b}}{r_{a b}^{n}}\right],
\end{aligned}
$$

where $a$ and $b$ are sites belonging to monomers $A$ and $B$, respectively, and $r_{a b}$ is the distance between two such sites. Each molecule features eight sites, both atomic and off atomic, described in detail in Ref. 12. The term containing the site charges $q_{a}$ and $q_{b}$ reproduces the asymptotics of the electrostatic component of the interaction energy, whereas the terms with coefficients $C_{n}^{a b}$ are responsible for the asymptotic behavior of the sum of induction and dispersion energies. Both these classes of terms are damped at short distances using the Tang-Toennies damping functions ${ }^{22}$ $f_{n}(\delta, r)=1-e^{-\delta r} \sum_{m=0}^{n}(\delta r)^{m} / m$ !. The fitting procedure consists, as in Ref. 12, of three stages. First, the charges $q_{a}$ are obtained from a linear fit to multipole moments of the water molecule, computed through $l=7$ with the KS determinant. Second, the dispersion-induction coefficients $C_{n}^{a b}$ are fitted to the sum of asymptotic dispersion and induction energies obtained on a grid of geometries using the one-center asymptotic expansion with van der Waals constants calculated from the $\mathrm{KS}$ multipoles and the FDDSs at the CKS level. During this fit, it is ensured (by imposing a constraint) that the overall $C_{6}$ asymptotic coefficient, represented by $C_{6}^{\mathrm{OO}}+4\left(C_{6}^{\mathrm{OH}}+C_{6}^{\mathrm{HH}}\right)$, is equal to the actual $a b$ initio value of $736.885 \AA^{6} \mathrm{kcal} / \mathrm{mol}$. Finally, the remaining parameters in Eq. (2) are least-square fitted to the 2510 SAPT(DFT) energies, with the charges and the $C_{n}^{a b}$ coefficients kept constant to ensure the correct asymptotics of the fit. It should be emphasized that the individual terms in Eq. (2) are physically interpretable only in the region of large intermolecular distances. For separations close to the minimum, only the sum of all these terms is meaningful. Overall, the fit features 77 parameters and has a rms error of $0.45 \mathrm{kcal} / \mathrm{mol}$, calculated for all 2510 data points. For the 1077 configurations in the well (i.e., $E_{\text {int }}^{\text {SAPT(DFT) }}<0$ ), the rms error is $0.1 \mathrm{kcal} / \mathrm{mol}$. To emphasize the origin of the $a b$ initio data and the functional similarity to the SAPT- $5 s$ potential of Ref. 12 , we will refer to the new fit as SDFT- $5 s$. The fit parameters are available from the authors upon request.

\section{STATIONARY POINTS}

Explorations of the SDFT-5s potential surface resulted in ten stationary points analogous to structures 1-10 considered in Ref. 23. We refer the reader to Fig. 1 in the latter work for schematic graphical representations of these structures. The global minimum is a nonplanar open structure of $C_{s}$ symmetry, containing a single hydrogen bond. The geometric parameters and energy of the minimum structure are reported in Table I, along with the corresponding theoretical results obtained in the literature and the experimental estimates. The SDFT-5 $s$ geometries agree very well with the full $a b$ initio optimization of Ref. 23, performed on the counterpoiseuncorrected coupled cluster singles and doubles with noniterative inclusion of triple excitations $[\operatorname{CCSD}(\mathrm{T})]$ potential 


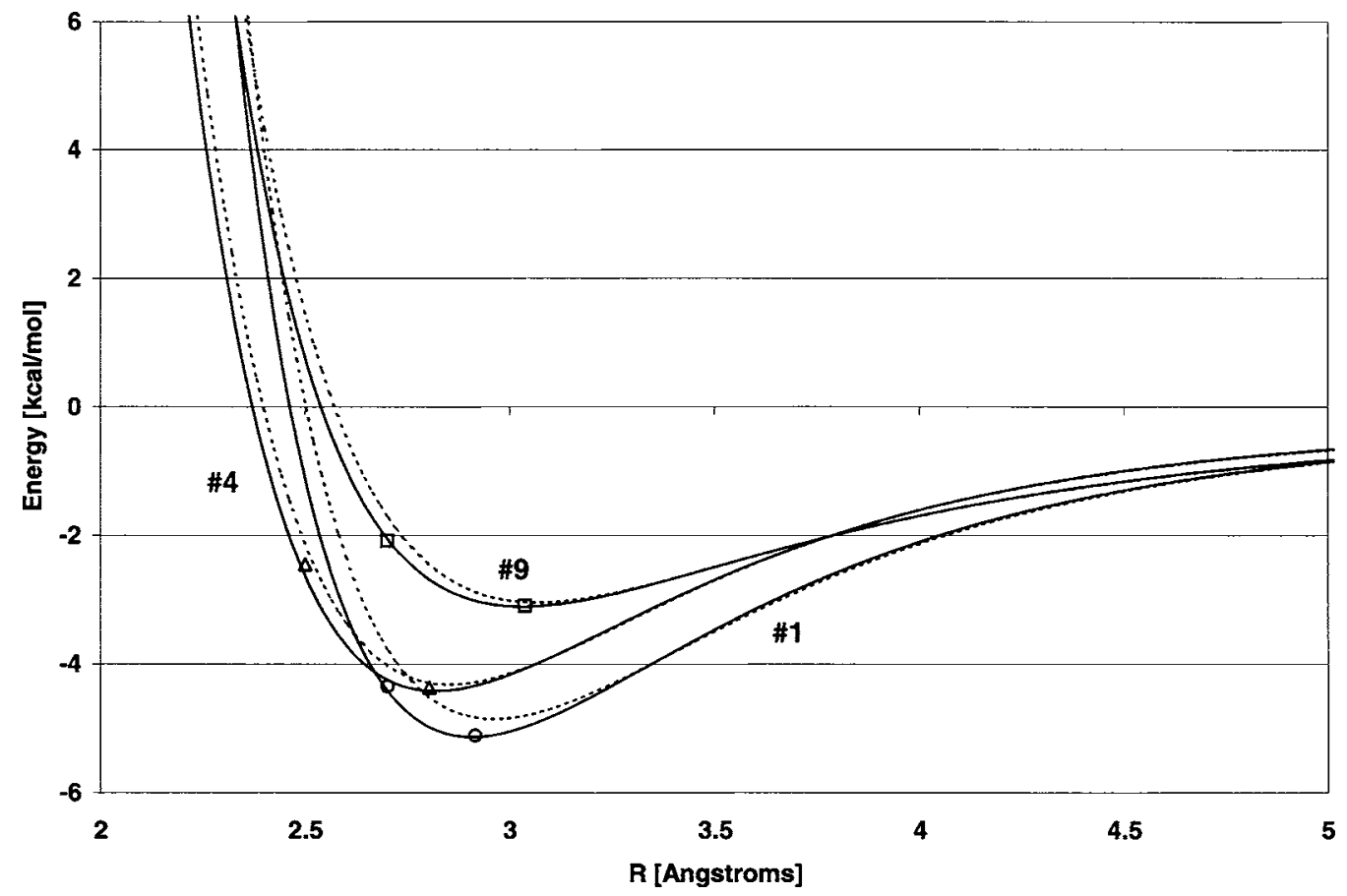

FIG. 1. Radial cross sections through the SDFT- $5 s$ (solid line) and SAPT-5s (dashed line) potentials for angular configurations close to the three stationary points of the dimer. $R$ is the distance between the centers of mass of the monomers. Circles, triangles, and squares show the extrapolated CCSD(T) results (see text) for structures 1,4 , and 9 , respectively.

surface in triple-zeta-quality basis set. These geometries also agree well with the two-dimensional optimization of Klopper et al. ${ }^{24}$ done using the $\operatorname{CCSD}(\mathrm{T})$ calculations extrapolated to the complete basis set (CBS) limit. The comparison is somewhat obscured by the fact that both these $\operatorname{CCSD}(\mathrm{T})$ optimizations included monomer relaxation effects. The SDFT-5s potential and both $\operatorname{CCSD}(\mathrm{T})$-based optimizations give the $\mathrm{O}-\mathrm{O}$ distance by about $0.05 \AA$ shorter than SAPT- $5 \mathrm{~s}$, closer to the mixed experimental-theoretical estimate of Ref. 12 . The angular coordinates from all the methods agree quite well with each other and with experiment. The minimum of

TABLE I. Parameters of the global minimum of water dimer. Distances in $\AA$, angles in degrees, and energies in $\mathrm{kcal} / \mathrm{mol} . r_{\mathrm{O}_{1} \mathrm{O}_{2}}$ is the distance between the oxygen atoms, $\theta_{\mathrm{O}_{2} \mathrm{O}_{1} \mathrm{H}_{2}}$ describes the deviation of hydrogen bond from linearity, and $\theta_{\mathrm{O}_{2} \mathrm{O}_{2} \text { bisec }}$ is the angle the acceptor plane makes with the $\mathrm{O}-\mathrm{O}$ line. See Fig. 1 in Ref. 23 for details.

\begin{tabular}{llccl}
\hline \hline & \multicolumn{1}{c}{$r_{\mathrm{O}_{1} \mathrm{O}_{2}}$} & $\theta_{\mathrm{O}_{2} \mathrm{O}_{1} \mathrm{H}_{2}}$ & $\theta_{\mathrm{O}_{1} \mathrm{O}_{2} \text { bisec }}$ & \multicolumn{1}{c}{ Energy } \\
\hline SDFT-5 $s$ & 2.9039 & 5.52 & 125.96 & -5.137 \\
$\mathrm{CCSD}(\mathrm{T})^{\mathrm{a}}$ & 2.909 & 4.47 & 124.92 & -5.02 \\
$\mathrm{CCSD}(\mathrm{T})^{\mathrm{b}}$ & $2.912 \pm 0.005$ & 5.5 & 124.4 & $-5.02 \pm 0.05$ \\
$\mathrm{SAPT}-5 s^{\mathrm{c}}$ & 2.9549 & 6.36 & 127.16 & -4.861 \\
Experiment $^{\mathrm{a}}$ & $2.91 \pm 0.005^{\mathrm{d}}$ & $-1 \pm 10^{\mathrm{e}}$ & $123 \pm 10^{\mathrm{e}}$ & $-5.00 \pm 0.7^{\mathrm{d}}$ \\
\hline \hline
\end{tabular}

${ }^{\mathrm{a}}$ Tschumper et al. (Ref. 23); CP-uncorrected optimization using CCSD(T) method in $\operatorname{TZ2P}(f, d)+$ dif basis set. Energy computed at the $\operatorname{CCSD}(\mathrm{T})$ $+\delta E_{\mathrm{BD}(\mathrm{TQ})}$ level with CBS-extrapolated MBPT2 component, where $\mathrm{BD}(\mathrm{TQ})$ denotes the Brueckner double method with noniterative triple and quadruple excitation.

${ }^{\mathrm{b}}$ Klopper et al. (Ref. 24); two-dimensional optimization on the CBSextrapolated $\operatorname{CCSD}(\mathrm{T})$ surface.

${ }^{\mathrm{c}}$ Mas et al. (Ref. 12); SAPT-5s fit to 2510 energies computed using SAPT method.

${ }^{\mathrm{d}}$ Mixed experimental-theoretical estimate from Ref. 12 .

${ }^{\mathrm{e} O d u t o l a}$ and Dyke (Ref. 25). the SDFT- $5 s$ potential is by $0.1 \mathrm{kcal} / \mathrm{mol}$ deeper than those predicted by the $a b$ initio $\operatorname{CCSD}(\mathrm{T})$ optimizations, ${ }^{23,24}$ although the monomer relaxation effects present in the latter results again make the comparison somewhat ambiguous. Since the intermolecular potential is very flat compared to the intramonomer potential, the fully optimized geometries have monomers very close to their isolated equilibrium geometries. In contrast, our calculation uses monomers in the vibrationally averaged geometry which leads to the interaction energies about $0.1 \mathrm{kcal} / \mathrm{mol}$ deeper around the minimum. To facilitate a better comparison between the SDFT- $5 s$ predictions and the $\operatorname{CCSD}(\mathrm{T})$ results, we performed the $\operatorname{CCSD}(\mathrm{T})$ calculations at the SDFT- $5 s$ global minimum geometry using the aug-cc-pVTZ and aug-cc-pVQZ basis sets of Kendall et $a l^{26}$ and the set of 14 bond functions of Ref. 15 , followed by an extrapolation to the CBS limit using the $X^{-3}$ scheme. Indeed, our extrapolated $\operatorname{CCSD}(\mathrm{T})$ interaction energy, equal to $-5.106 \mathrm{kcal} / \mathrm{mol}$, differs from the SDFT- $5 \mathrm{~s}$ one quoted in Table I only by $0.03 \mathrm{kcal} / \mathrm{mol}$. Based on the results of Refs. 23 and 24 , our $\operatorname{CCSD}(\mathrm{T})$ extrapolation is most likely accurate to within a few hundredths of $\mathrm{kcal} / \mathrm{mol}$. Their estimation of accuracy suggests in fact that the exact result for this geometry is larger in magnitude, and therefore even closer to the SAPT(DFT) value. Note, however, that this startling agreement may deteriorate somewhat if SAPT(DFT) calculations are performed in a larger basis. Overall, the SDFT- $5 s$ potential predicts the well depth more accurately than SAPT $-5 s$, which is by about $0.2 \mathrm{kcal} / \mathrm{mol}$ too shallow.

The relationship between the SDFT- $5 s$ and SAPT- $5 s$ potentials can be further assessed from Fig. 1, where the radial cross sections are plotted for configurations close to the three 
TABLE II. Interaction energies (in $\mathrm{cm}^{-1}$ ) at stationary-point structures of water dimer relative to the minimum. See Fig. 1 in Ref. 23 for graphical representations of all structures. Unless noted otherwise, the index is the same for all calculations.

\begin{tabular}{lcccr}
\hline \hline \multicolumn{1}{c}{$\begin{array}{c}\text { Structure } \\
\text { No. }\end{array}$} & Index & SDFT-5s & $\begin{array}{r}\text { SAPT-5s } \\
(\text { Ref. 12) }\end{array}$ & $\begin{array}{r}\text { CCSD }(\mathrm{T}) \\
\text { (Ref. 23) }\end{array}$ \\
\hline $2\left(\right.$ open $\left.C_{1}\right)$ & 1 & 180 & $\ldots$ & $181 \pm 5$ \\
$3\left(\right.$ planar open $\left.C_{s}\right)$ & $2^{\mathrm{a}}$ & 181 & 156 & $198 \pm 6$ \\
$4\left(\right.$ cyclic $\left.C_{i}\right)$ & 1 & 251 & 185 & $245 \pm 15$ \\
$5\left(\right.$ cyclic $\left.C_{2}\right)$ & $1^{\mathrm{b}}$ & 360 & $\ldots$ & $333 \pm 15$ \\
$6\left(\right.$ cyclic $\left.C_{2 h}\right)$ & 3 & 378 & 280 & $348 \pm 18$ \\
7 (triply H-bonded $\left.C_{s}\right)$ & 2 & 741 & 659 & $634 \pm 18$ \\
$8\left(\right.$ double bifurcated $\left.C_{2 h}\right)$ & 3 & 1325 & 1244 & $1249 \pm 21$ \\
9 (nonplanar bifurcated $\left.C_{2 v}\right)$ & 1 & 710 & 636 & $625 \pm 16$ \\
$10\left(\right.$ planar bifurcated $\left.C_{2 v}\right)$ & 2 & 978 & 897 & $948 \pm 23$ \\
\hline
\end{tabular}

Saddle point of index 1 on SAPT- $5 s$ surface.

${ }^{\mathrm{b}}$ Stationary point of index 2 on $\operatorname{CCSD}(\mathrm{T})$ surface.

stationary points of the dimer. To put both sets of curves in context, we performed several single-point $\operatorname{CCSD}(\mathrm{T})$ calculations extrapolated to the CBS limit as described above for the global minimum. The results of these calculations may be considered benchmarks in our comparison of the SDFT-5s and SAPT-5s surfaces. From Fig. 1, one can see that the extrapolated $\operatorname{CCSD}(\mathrm{T})$ energies are, in fact, very close to the SDFT- $5 s$ curves, both near the minima and on the repulsive wall, suggesting that SDFT-5 $s$ is quantitatively correct and more accurate than SAPT- $5 s$. The latter potential is too shallow and the onset of the repulsive wall occurs for intermolecular distances larger than those predicted by SDFT- $5 s$ and by the $\operatorname{CCSD}(\mathrm{T})$ benchmark. The consequence of this is that the minima of SAPT- $5 s$ (including the global minimum, structure 1) are located too far outward. Moreover, the accuracy of SAPT- $5 s$ is rather uneven for different configurations, curve 9 being closer (at least in the vicinity of the minimum) to its SDFT- $5 s$ counterpart than curve 1 or 4.

As in the case of the global minimum, geometries of stationary points $2-10$ resulting from the SDFT- $5 s$ potential are closer to those obtained using the $\operatorname{CCSD}(\mathrm{T}) a b$ initio optimizations $^{23}$ than to the SAPT- $5 s$ ones. ${ }^{12} \mathrm{We}$ calculated the geometric parameters of these structures, as defined in Ref. 23, and found that these parameters differ by no more than a few hundredths of angstroms and by a few degrees from the values reported in Table III of Ref. 23. In particular, as shown in Table II, SDFT- $5 s$ predicts the lowest saddle point to be a semiplanar, $\mathrm{H}$-bonded structure of $C_{1}$ symmetry (2), consistently with predictions of the $\operatorname{CCSD}(\mathrm{T})$ optimizations. ${ }^{23}$ On the SAPT- $5 s$ surface, structure 2 is missing and a planar geometry similar to 3 is the lowest saddle point. The SAPT- $5 s$ potential also does not predict structure 5 , whereas such a structure is found on the SDFT-5 $s$ and the $\operatorname{CCSD}(\mathrm{T})$ surfaces. With just two exceptions, the indices (i.e., the numbers of negative Hessian eigenvalues) are consistent between different potential surfaces. Table II shows the barrier heights associated with the stationary points, i.e., the interaction energies of all structures relative to the respective global minima. These barriers are important from the point of view of dimer dynamics since they influence the tunneling splittings and shifts of the energy levels. Barriers
2-6 from SDFT-5 $s$ are significantly higher than those from the SAPT- $5 s$ surface, and agree very well with the results of the $\operatorname{CCSD}(\mathrm{T})$ optimizations, ${ }^{23}$ whereas barriers 7-9 of the latter optimizations agree best with the SAPT- $5 s$ results. It should be stressed, however, that the barriers computed in Ref. 23 contain monomer relaxation effects and therefore cannot be precisely compared to the ones derived from rigid potentials.

\section{SECOND VIRIAL COEFFICIENT}

One of the ways to evaluate the quality of a dimer interaction potential is to compute the second virial coefficient $B(T)$ and compare it to experimental results. Due to the relatively small moments of inertia of the water molecule, any theoretical calculation of $B(T)$ for water must account for quantum effects, which turn out to be visible even for temperatures as high as $1000 \mathrm{~K}^{12}$ In the treatment of quantum effects, we follow Schenter, ${ }^{27}$ who suggested the use of the effective two-body potential of the form

$$
V_{\mathrm{eff}}=V+\frac{\hbar^{2}}{24\left(k_{B} T\right)^{2}} \sum_{i=1,2}\left[\frac{F_{i}^{2}}{M}+\sum_{\alpha} \frac{T_{\alpha i}^{2}}{I_{\alpha}}\right],
$$

where $V$ is the regular pair potential [computed here from Eq. (2)], $k_{B}$ is the Boltzmann constant, $\mathbf{F}_{i}, i=1,2$ is the force on molecule $i$ exerted by the partner, and $T_{\alpha i}, i=1,2, \alpha$ $=1,2,3$, is the component of the torque on molecule $i$ along the principal axis $\alpha$ of this molecule. The mass of the molecule and its principal moments of inertia are denoted by $M$ and $I_{\alpha}, \alpha=1,2,3$, respectively. The second virial coefficient (for rigid monomers) is then given by a classical expression with the usual two-body potential replaced by $V_{\text {eff }}$ :

$$
B(T)=-\frac{1}{2} \int d \mathbf{R}\left\langle e^{-V_{\text {eff }} / k_{B} T}-1\right\rangle_{\Omega_{1} \Omega_{2}} .
$$

In the equation above, $\mathbf{R}$ is the vector connecting the centers of mass of the two monomers, $\Omega_{1}$ and $\Omega_{2}$ are two sets of Euler angles (in a laboratory frame) describing the monomers' orientations, and the shorthand notation \langle\rangle$_{\Omega_{1} \Omega_{2}}$ $\equiv\left[1 /\left(8 \pi^{2}\right)^{2}\right] \int d \Omega_{1} d \Omega_{2}$ is used for angular averaging. As argued in Ref. 27, approximation (4) gives the second virial coefficient within a few percent of the fully quantum pathintegral Monte Carlo calculations.

The values of $B(T)$ were calculated over a wide range of temperatures using the integration technique described in Ref. 15. The results obtained with the SDFT-5s and SAPT-5s potentials are shown in Table III and compared to experimental data. For temperatures below $600 \mathrm{~K}$, the SDFT- $5 s$ values are more negative than the SAPT- $5 s$ ones and agree with experiment much better. This behavior is consistent with the well of the SDFT- $5 s$ potential being deeper than that of SAPT- $5 s$. In the high-temperature regime, the SDFT- $5 s$ results are slightly below experiment and the SAPT- $5 s$ potential seems to offer the best agreement. This, however, may be the consequence of the fact that the theoretical $B(T)$ values derived from SAPT- $5 s$ were actually included as hightemperature data points in the correlation procedure of Ref. 28. When comparing the theoretical and experimental $B(T)$ values from Table III, one also needs to remember that the 
TABLE III. Second virial coefficient of water (in $\mathrm{cm}^{3} / \mathrm{mol}$ ). Errors of numerical integration are below $0.5 \%$ for $T<1000 \mathrm{~K}$ and below $0.3 \mathrm{~cm}^{3} / \mathrm{mol}$ for $T>1000 \mathrm{~K}$.

\begin{tabular}{crrr}
\hline \hline$T(\mathrm{~K})$ & SDFT-5s & ${\text { SAPT- } 5 s^{\mathrm{a}}}$ & Expt. $^{\mathrm{b}}$ \\
\hline 273.15 & -1827.1 & -1553.4 & -1916.9 \\
293.15 & -1270.8 & -1101.7 & -1307.8 \\
295.15 & -1229.3 & -1067.6 & -1262.9 \\
298.15 & -1170.8 & -1019.3 & -1199.7 \\
323.15 & -811.1 & -719.3 & -816.7 \\
373.15 & -456.6 & -415.4 & -451.6 \\
423.15 & -294.8 & -272.5 & -289.9 \\
448.15 & -245.1 & -227.9 & -240.6 \\
473.15 & -207.3 & -193.6 & -203.2 \\
523.15 & -154.1 & -144.8 & -150.5 \\
573.15 & -119.0 & -112.4 & -115.7 \\
673.15 & -76.5 & -72.5 & -73.6 \\
773.15 & -52.2 & -49.4 & -49.7 \\
873.15 & -36.8 & -34.7 & -34.6 \\
973.15 & -26.2 & -24.5 & -24.4 \\
1000 & -23.9 & -22.3 & -22.2 \\
1500 & -1.7 & -0.7 & -0.8 \\
2000 & 6.2 & 7.0 & 6.9 \\
2500 & 10.0 & 10.6 & 10.6 \\
3000 & 12.0 & 12.6 & 12.6 \\
\hline \hline
\end{tabular}

${ }^{\mathrm{a}}$ Calculated in the present work from the potential of Ref. 12

${ }^{\mathrm{b}}$ Correlation of experimental data by Harvey and Lemmon (Ref. 28).

former results have been obtained within the rigid-monomer approximation. As pointed out in Refs. 28 and 29, monomer flexibility effects may become important in both the lowtemperature and high-temperature regimes. Studies performed within the classical approximation showed ${ }^{30}$ that these effects can make $B(T)$ more negative by as much as $10 \%$. Although in our rigid-monomer calculations a part of the flexibility effect is implicitly taken into account by using the vibrationally averaged monomer geometry, some negative contribution may still be expected from a fully quantum calculation with flexible monomers. In such a case, the values of $B(T)$ predicted by the SDFT-5s potential would have to be considered somewhat too negative compared to the rigid-monomer accuracy limit. Nevertheless, the ability of this potential to reproduce the second virial coefficient in such good agreement with experiment is yet another confirmation of the effectiveness of the SAPT(DFT) methodology.

\section{VIBRATION-ROTATION-TUNNELING SPECTRUM}

In first approximation, the water dimer may be considered a semirigid prolate symmetric rotor with energy levels characterized by the total angular momentum quantum number $J$ and its projection $K$ onto the principal axis corresponding to the largest rotational constant $A$ (this axis is approximately parallel to the vector connecting the two monomers). The rotational constants of such a rotor depend on its vibrational state. In the ground vibrational state, the dimer may be thought of as oscillating around the global minimum configuration, or, more precisely, tunneling between eight such symmetry-equivalent configurations, separated by relatively low barriers (see Table II). As a result of this tunneling, the ground vibrational state for fixed $J$ and $K$ is split into eight quasidegenerate states grouped into six energy levels. The higher vibrational states undergo analogous splittings. These six levels are further subdivided in two three-level subgroups, separated by a relatively large splitting denoted by $a(K)$ to emphasize its dependence on the $K$ quantum number. This large splitting is a result of the so-called acceptor tunneling through the lowest barrier 2 (or 3 in the case of SAPT$5 s$ ). Other tunneling processes, through barriers 4 and 9 , result in the so-called interchange splittings $i_{1}$ and $i_{2}$ between the highest and the lowest level of each subgroup.

The magnitudes of all tunneling splittings and the positions $o_{1}$ and $o_{2}$ of the subgroup origins are accurately measured by microwave and far-infrared spectroscopy (see Ref. 13 for a review of experimental work). The ability to reproduce these subtle features of the dimer spectrum is a very stringent criterion for the evaluation of any theoretically derived water dimer potential. To test the SDFT- $5 s$ surface in this context, we performed fully quantum, six-dimensional calculations of the dimer energy levels. The parameters of the ground-state tunneling splittings mentioned above and the dimer rotational constants obtained from the calculations are shown in Table IV. Precise definitions of all quantities as well as the detailed description of the computational method may be found in Ref. 13, where analogous calculations have been reported for the SAPT-5s surface. The results of the latter calculations are also shown in Table IV for comparison, along with those obtained from other theoretically derived literature potentials.

The end-over-end rotational constant $B+C$ resulting from SDFT- $5 s$ is by $0.1 \mathrm{~cm}^{-1}$ larger than the one obtained with the SAPT-5 $s$ potential and agrees with experiment slightly worse. This indicates that, on the average, the intermolecular distance predicted by SDFT- $5 s$ is somewhat too small. On the other hand, the $A$ rotational constant from SDFT- $5 s$ is closer to experiment than the SAPT- $5 s$ one, suggesting that the angular degrees of freedom in the vicinity of the global minimum are modeled better by the former potential. The $A$ constant and the positions $o_{1}$ and $o_{2}$ of the $J=K$ $=1$ levels come out best from the anisotropic site potential(ASP-) S potential. ${ }^{31}$ The $o_{1}$ and $o_{2}$ values from SDFT-5s and SAPT- $5 s$ are $10 \%-15 \%$ too large, but still better than the values produced by the remaining two potentials from Table IV. The acceptor splittings $a(K=0)$ and $a(K=1)$ are not measured separately and only their sum is known. This sum is best reproduced by the ASP-S potential, whereas SAPT-5s gives a value which is $44 \%$ too large. SDFT- $5 s$ represents an improvement over SAPT-5s, reducing the discrepancy to $28 \%$. This reduction of the acceptor splitting is consistent with the barrier 2 predicted by SDFT- $5 s$ being higher than the barrier 3 of SAPT- $5 s$. The other two potentials give acceptor splittings too small or too large by a factor of about 2 . SAPT- $5 s$ excels in reproducing the small interchange splittings $i_{1}$ and $i_{2}$. The results from SDFT- $5 s$, smaller than the experimental values on the average by a factor of 1.7 , are still better than their counterparts produced by the remaining potentials, which are too large or too small by at least a factor of 2, often much worse. Overall, the dimer VRT spectrum obtained from the SDFT-5s potential is of much better quality than spectra obtained from most other theoretically 
TABLE IV. Rotational constants and tunneling splittings $\left(\right.$ in $\left.\mathrm{cm}^{-1}\right)$ for the ground vibrational state of $\left(\mathrm{H}_{2} \mathrm{O}\right)_{2}$. See Ref. 13 for definitions, computational details, and sources of experimental data.

\begin{tabular}{lcccccc}
\hline \hline & SDFT-5 $s$ & ${\text { SAPT- } 5 s^{\mathrm{a}}}$ & ASP-W $^{\mathrm{b}}$ & ASP-S $^{\mathrm{b}}$ & MCY-KW $^{\mathrm{c}}$ & Expt. \\
\hline$B+C$ & 0.422 & 0.409 & 0.438 & 0.390 & 0.420 & 0.411 \\
$A$ & 7.21 & 6.93 & 8.05 & 7.84 & 6.50 & 7.59 \\
$J=K=0$ & & & & & & \\
$a(K=0)$ & 14.26 & 16.19 & 4.67 & 10.01 & 18.81 & $\ldots$ \\
$i_{1}$ & 0.44 & 0.75 & 3.44 & 1.75 & 0.04 & 0.75 \\
$i_{2}$ & 0.42 & 0.67 & 3.45 & 1.53 & 0.04 & 0.65 \\
$J=K=1$ & & & & & & \\
$o_{1}$ & 15.91 & 16.77 & 10.71 & 13.76 & 18.03 & 14.39 \\
$o_{2}$ & 12.35 & 12.87 & 9.61 & 11.54 & 13.36 & 11.66 \\
$a(K=1)$ & 3.56 & 3.90 & 1.11 & 2.23 & 4.67 & $\cdots$ \\
$i_{1}$ & 0.43 & 0.73 & 3.38 & 1.63 & 0.03 & 0.70 \\
$i_{2}$ & 0.32 & 0.53 & 2.89 & 1.26 & 0.02 & 0.54 \\
$a(K=0)+a(K=1)$ & 17.82 & 20.09 & 5.77 & 12.24 & 23.48 & 13.92 \\
\hline \hline
\end{tabular}

${ }^{\mathrm{a}}$ Calculations of Ref. 13 with the potential of Ref. 12 .

${ }^{\mathrm{b}}$ Computed from energy levels obtained in Ref. 33 with the potential of Ref. 31 .

${ }^{\mathrm{c}}$ Computed from energy levels obtained in Ref. 33 with the potential of Ref. 34.

derived potentials, including ASP-W and MCY-KW shown here. The SDFT- $5 s$ spectrum is comparable to those predicted by the SAPT- $5 s$ and ASP-S potentials, with some features being reproduced better by the SDFT- $5 s$ potential.

\section{SIMULATIONS OF AMBIENT WATER}

A pair potential, whether fitted to $a b$ initio dimer interaction energies or tuned to dimer experimental data, cannot succeed in describing larger clusters or condensed phases of water due to a large contribution of nonadditive effects in such systems. Thus, to test the performance of SDFT- $5 s$ potential in predicting the properties of liquid water, one needs to add at least the three-body, and possibly also higher-order many-body terms in the potential. Actually, the convergence of such a many-body expansion is by itself an interesting problem. In Ref. 14, this issue was investigated using SAPT$5 s$ as the underlying pair potential. Here, we shall reexamine the problem using SDFT- $5 s$ surface, which, as suggested by the analysis of Sec. III, is overall somewhat more accurate than SAPT-5s.

Following Ref. 14, we consider several many-body potential models. In the model called just SDFT- $5 s$, the potential energy of a system of $N$ molecules will be assumed as a sum over pairs,

$$
U_{2 B}=\sum_{A<B}^{N} V(A, B),
$$

where $V(A, B)$ is the SDFT-5s potential function of Eq. (2), evaluated for molecules $A$ and $B$. In the model referred to as SDFT- $5 s+3 \mathrm{~B}$, we utilize the three-body nonadditive potential developed in Ref. 32. Denoting such a potential evaluated for a triple of molecules $A, B$, and $C$ as $V_{3}(A, B, C)$, we can write the total energy of $N$ molecules as

$$
U_{3 \mathrm{~B}}=U_{2 \mathrm{~B}}+\sum_{A<B<C}^{N} V_{3}(A, B, C)
$$

To test the importance of higher many-body effects, we approximate these effects using a simple, one-center polarization model, defined by Eqs. (9)-(12) of Ref. 32, which accounts for the leading components of the induction nonadditivity. The interaction energy in the resulting model, SDFT $-5 s+\mathrm{NB}$, is given by

$$
\begin{aligned}
U_{\mathrm{NB}}= & U_{3 \mathrm{~B}}+V_{N}^{\text {ind }}-\sum_{A<B} V_{2}^{\text {ind }}(A, B)-\sum_{A<B<C}\left[V_{3}^{\text {ind }}(A, B, C)\right. \\
& \left.-V_{2}^{\text {ind }}(A, B)-V_{2}^{\text {ind }}(A, C)-V_{2}^{\text {ind }}(B, C)\right]
\end{aligned}
$$

where the classical induction energy $V_{m}^{\text {ind }}$ is evaluated from Eq. (9) of Ref. 32 (denoted there by $E_{\mathrm{pol}}$ ) for a given subset of $m$ molecules out of $N$. Note that the double and the triple sum on the right hand side of Eq. (7) are the two-body and three-body components, respectively, of the total $N$-body induction energy $V_{N}^{\text {ind }}$. These components are subtracted from $V_{N}^{\text {ind }}$ to avoid double counting of the two- and three-body induction effects, already present in $U_{3 \mathrm{~B}}$. The models introduced above closely correspond to the models SAPT $-5 s$, SAPT $-5 s+3 \mathrm{~B}$, and SAPT $-5 s+\mathrm{NB}$ used in simulations of Ref. 14.

The interaction models described above have been used in molecular dynamics (MD) simulations at ambient conditions, i.e., $T=298.15 \mathrm{~K}$ and density $\rho=1 \mathrm{~g} / \mathrm{cm}^{3}$, with 128 molecules in a cubic box with periodic boundary conditions. The target temperature was approximately achieved by means of velocity scaling in the equilibration stage, which lasted for about 5 ps. Averages were collected for another 10-20 ps in the NVE ensemble. The cut-off distance was chosen as half of the length of the box, i.e., 7.82 A. A Ewald summation was used to evaluate the long-range electrostatics, whereas the short-range interactions were smoothly damped to zero around the cut-off distance to avoid the total energy drift. 

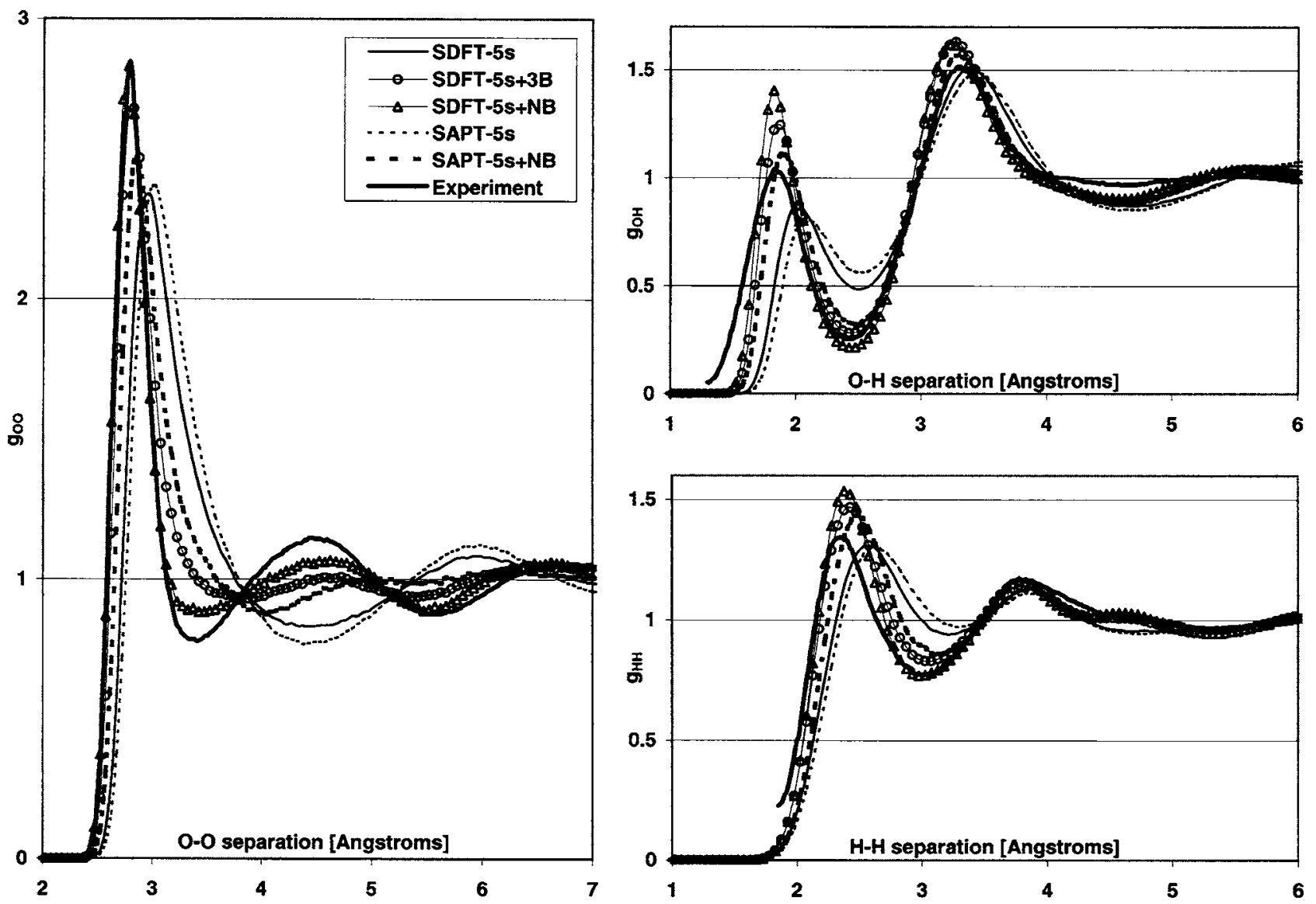

FIG. 2. Atom-atom radial distribution functions from different potential models. The SAPT- $5 s$ and SAPT- $5 s+$ NB curves are taken from Ref. 14 and the experimental curves from Soper's work (Ref. 35).

From the point of view of many-body convergence, the structure of liquid water, represented by the atom-atom radial distribution functions (RDFs), is of special interest. This issue was studied in Ref. 14 using MC simulations based on the SAPT- $5 s$ pair potential. The RDFs resulting from the SAPT- $5 s$ and SAPT- $5 s+$ NB simulations of Ref. 14 (defined by the potentials $U_{2 \mathrm{~B}}$ and $U_{\mathrm{NB}}$, respectively, with the twobody part given by the SAPT- $5 s$ potential) are plotted in Fig. 2 for comparison with the present results from the simulations based on the SDFT- $5 s$ potential.

In the absence of nonadditive effects, liquid structures obtained from both pair potentials (the curves SAPT- $5 s$ and SDFT-5s) are very similar, with both $g_{\mathrm{OO}}$ functions featuring a very broad first peak around $3.0 \AA$ and a broad first minimum around $4.5 \AA$, much farther than the experimental minimum. The peak of the SAPT- $5 s(2 \mathrm{~B}) g_{\text {OO }}$ curve is shifted by about $0.05 \AA$ to the right with respect to the SDFT- $5 s(2 \mathrm{~B})$ one, consistently with the $\mathrm{O}-\mathrm{O}$ distance in the dimer minimum being larger in the case of SAPT- $5 s$ (see Table I). Integrating each two-body $g_{\mathrm{OO}}$ function up to the first minimum gives a coordination number of about 12 , indicating a rare-gas like arrangement of oxygen atoms rather than the expected tetrahedral structure. The first peak on the $g_{\mathrm{OH}}$ curves from both two-body simulations is strongly suppressed and shifted to the right, indicating an insufficient (compared to experiment) number of hydrogen bonds. The $g_{\mathrm{HH}}$ two-body curves agree better with experiment, but even here differences are substantial. The shapes and positions of all features greatly improve when the nonadditive interactions are added to either pair potential. The SDFT- $5 s+3 \mathrm{~B}$ $g_{\text {OO }}$ curve has the main peak shifted to the experimental position, and the first minimum is also significantly moved to the left. Addition of $N>$ three-body induction interactions in the SDFT- $5 s+$ NB simulation deepens the first minimum and shifts it further to the left, which indicates the onset of tetrahedral structure and leads to generally good agreement with experiment, giving a coordination number equal to 5 . In contrast, the SAPT $-5 s+\mathrm{NB}$ simulation produces a still insufficiently structured liquid, with the oxygen-oxygen coordination number close to 8 . Moreover, the SAPT $-5 s+\mathrm{NB} g_{\mathrm{OO}}$ curve is very similar ${ }^{14}$ to the SAPT- $5 s+3 \mathrm{~B}$ one (not shown for clarity), which led to the conclusion in Ref. 14 that the many-body nonadditive effects with $N>3$ are not important for saturation of the liquid structure. As seen from the SDFT$5 s+3 \mathrm{~B}$ and SDFT $-5 s+\mathrm{NB} g_{\mathrm{OO}}$ curves, the use of a different pair potential indicates that these effects are relatively important. One could conclude that the better performance of the SDFT- $5 s$ potential compared to SAPT- $5 s$ is due to its greater depth. Such a conclusion would be incorrect as scaling of SAPT- $5 s$ does not improve the simulation results. The key to understanding the differences in the results of simulations with the two potentials is the uneven accuracy of SAPT- $5 s$ pointed out in Sec. III. As seen from Fig. 1, the SAPT-5s surface underestimates the attraction at the H-bonded configurations like 1, characteristic for tetrahedral structure, whereas for other geometries the SAPT- $5 s$ potential is rela- 
tively more accurate. This results in an insufficient preference for locking of the molecules in tetrahedral geometries under the influence of many-body forces.

\section{CONCLUSIONS}

Symmetry-adapted perturbation theory with DFT description of intramonomer correlation has been used to obtain a new, six-dimensional interaction potential for water dimer. The potential, referred to as SDFT- $5 s$, is represented by a site-site formula fitted to interaction energies computed at 2510 dimer geometries. Comparison of stationary points found on the SDFT- $5 s$ surface with the results of $a b$ initio optimizations at the $\operatorname{CCSD}(\mathrm{T})$ level, ${ }^{23}$ as well as with extrapolated $\operatorname{CCSD}(\mathrm{T})$ calculations, ${ }^{24}$ suggests that the new potential represents an overall improvement over the SAPT-5s potential of Ref. 12, especially for hydrogen-bonded configurations, abundant in liquid water. Although the latter potential performs better in reproducing the small interchange splittings in the dimer VRT spectra, SDFT- $5 s$ gives a more accurate value of the acceptor splitting. The second virial coefficient calculated with SDFT- $5 s$ is closer to experiment than that from SAPT-5s, especially for lower temperatures. Careful evaluation of monomer flexibility effects may be necessary to fully assess the accuracy of the calculated virial data. The SDTF- $5 s$ potential allowed to shed new light on the problem of convergence of many-body expansion of the interaction energy, bringing conclusions different from those based on the SAPT- $5 s$ potential. ${ }^{14}$ Simulations of ambient liquid water with pair interactions represented by SDFT-5s and the nonadditive interactions included as in Ref. 14 lead to a liquid structure in very good agreement with experiment, better than achieved with SAPT- $5 s$ potential. Contrary to the findings of Ref. 14, nonadditive interactions beyond threebody level turn out to be important for the formation of the tetrahedral structure of the liquid. Based on all these comparisons, it appears that SDFT- $5 s$ provides the best current characterization of the interactions between two water molecules.

The overall better performance of the SDFT- $5 s$ potential compared to the SAPT- $5 s$ potential provides a strong confirmation of the high reliability of the SAPT(DFT) method, demonstrated before in Refs. 10 and 11. One should point out that this better performance is mainly due to the faster convergence of SAPT(DFT) in the basis set size compared to SAPT, see Refs. 10 and 11 (recall that both SDFT-5s and SAPT- $5 s$ potentials were computed using the same basis set, the same set of grid points, and the same form of the fit). If SAPT calculations are performed in larger basis sets and extrapolated to the CBS limit, the results become very close to those of SAPT(DFT) in the current basis, to within $0.13 \mathrm{kcal} / \mathrm{mol}$; cf. Table V of Ref. 11.

\section{ACKNOWLEDGMENTS}

Funding for this work was provided by NSF Grant Nos. CHE-0239611 and CHE-0555979.

${ }^{1}$ H. L. Williams and C. F. Chabalowski, J. Phys. Chem. A 105, 646 (2001).

${ }^{2}$ A. J. Misquitta and K. Szalewicz, Chem. Phys. Lett. 357, 301 (2002).

${ }^{3}$ A. J. Misquitta, B. Jeziorski, and K. Szalewicz, Phys. Rev. Lett. 91, 033201 (2003).

${ }^{4}$ A. Hesselmann and G. Jansen, Chem. Phys. Lett. 357, 464 (2002).

${ }^{5}$ A. Hesselmann and G. Jansen, Chem. Phys. Lett. 362, 319 (2002).

${ }^{6}$ A. Hesselmann and G. Jansen, Chem. Phys. Lett. 367, 778 (2003).

${ }^{7}$ A. Hesselmann, G. Jansen, and M. Schütz, J. Chem. Phys. 122, 014103 (2005).

${ }^{8}$ R. Podeszwa, R. Bukowski, and K. Szalewicz, J. Chem. Theory Comput. 2, 400 (2006)

${ }^{9}$ R. Bukowski, R. Podeszwa, and K. Szalewicz, Chem. Phys. Lett. 414, 111 (2005).

${ }^{10}$ A. J. Misquitta and K. Szalewicz, J. Chem. Phys. 122, 214109 (2005).

${ }^{11}$ A. J. Misquitta, R. Podeszwa, B. Jeziorski, and K. Szalewicz, J. Chem. Phys. 123, 214103 (2005).

${ }^{12}$ E. M. Mas, R. Bukowski, K. Szalewicz, G. Groenenboom, P. E. S. Wormer, and A. van der Avoird, J. Chem. Phys. 113, 6687 (2000).

${ }^{13}$ G. C. Groenenboom, P. E. S. Wormer, A. van der Avoird, E. M. Mas, R. Bukowski, and K. Szalewicz, J. Chem. Phys. 113, 6702 (2000).

${ }^{14}$ E. M. Mas, R. Bukowski, and K. Szalewicz, J. Chem. Phys. 118, 4404 (2003).

${ }^{15}$ E. M. Mas, K. Szalewicz, R. Bukowski, and B. Jeziorski, J. Chem. Phys. 107, 4207 (1997).

${ }^{16}$ J. P. Perdew, K. Burke, and M. Ernzerhof, Phys. Rev. Lett. 77, 3865 (1996).

${ }^{17}$ C. Adamo and V. Barone, J. Chem. Phys. 110, 6158 (1999).

${ }^{18}$ E. Fermi and E. Amaldi, Mem. Accad. Italia 6, 119 (1934).

${ }^{19}$ D. J. Tozer and N. C. Handy, J. Chem. Phys. 109, 10180 (1998).

${ }^{20}$ S. G. Lias, NIST Chemistry WebBook, NIST Standard Reference Database Number 69 (http://webbook.nist.gov).

${ }^{21}$ R. D. Amos, I. L. Alberts, J. S. Andrews et al., CADPAC: The Cambridge analytic derivatives package: a suite of quantum chemistry programs, Issue 6, Cambridge, 1995.

${ }^{22}$ K. T. Tang and J. P. Toennies, J. Chem. Phys. 80, 3726 (1984).

${ }^{23}$ G. S. Tschumper, M. L. Leininger, B. C. Hoffman, E. F. Valeev, H. F. Schaefer III, and M. Quack, J. Chem. Phys. 116, 690 (2002).

${ }^{24}$ W. Klopper, J. G. C. M. van Duijneveldt-van de Rijdt, and F. B. van Duijneveldt, Phys. Chem. Chem. Phys. 22, 2227 (2000).

${ }^{25}$ J. A. Odutola and T. R. Dyke, J. Chem. Phys. 72, 5062 (1980).

${ }^{26}$ R. A. Kendall, T. H. Dunning, and R. J. Harrison, J. Chem. Phys. 96, 6796 (1992)

${ }^{27}$ G. K. Schenter, J. Chem. Phys. 117, 6573 (2002).

${ }^{28}$ A. H. Harvey and E. W. Lemmon, J. Phys. Chem. Ref. Data 33, 369 (2004).

${ }^{29}$ R. A. Bustos Marún, E. A. Coronado, and J. C. Ferrero, Chem. Phys. Lett. 405, 203 (2005)

${ }^{30}$ K. Refson, G. C. Lie, and E. Clementi, J. Chem. Phys. 87, 3634 (1987).

${ }^{31}$ C. Millot and A. J. Stone, Mol. Phys. 77, 439 (1992).

${ }^{32}$ E. M. Mas, R. Bukowski, and K. Szalewicz, J. Chem. Phys. 118, 4386 (2003).

${ }^{33}$ R. S. Fellers, L. B. Braly, R. J. Saykally, and C. Leforestier, J. Chem. Phys. 110, 6306 (1999).

${ }^{34}$ S. Kuwajima and A. Warshel, J. Phys. Chem. 94, 460 (1990).

${ }^{35}$ A. K. Soper, Chem. Phys. 258, 121 (2000). 